\title{
Efficient Somatic Embryogenesis and Organogenesis of Self-Pollination Artemisia annua Progeny and Artemisinin Formation in Regenerated Plants
}

\author{
Fatima Alejos-Gonzalez, Kelly Perkins, Malcolm Isaiah Winston, De-Yu Xie ${ }^{*}$
}

Department of Plant and Microbial Biology, North Carolina State University, Raleigh, USA.

Email: *dxie@ncsu.edu

Received September $23^{\text {rd }}, 2013$; revised October $20^{\text {th }}, 2013$; accepted October $26^{\text {th }}, 2013$

Copyright (C) 2013 Fatima Alejos-Gonzalez et al. This is an open access article distributed under the Creative Commons Attribution License, which permits unrestricted use, distribution, and reproduction in any medium, provided the original work is properly cited.

\begin{abstract}
To enhance the understanding of artemisinin biosynthesis, we have successfully bred self-pollination Artemisia annua plants. Here, we report efficient somatic embryogenesis and organogenesis of self-pollination plants and artemisinin formation in regenerated plants. The first through sixth nodal leaves of seedlings are used as explants. On agar-solidified MS basal medium supplemented with TDZ $(0.6 \mathrm{mg} / \mathrm{l})$ and IBA $(0.1 \mathrm{mg} / \mathrm{l})$, all explants after inoculation of less than 3 weeks start to form embryogenic calli, which further produce globular, torpedo, heart and early cotyledon embryos. In all six positional leaves, explants from the sixth leaf show the rapidest responses to induction of embryogenic calli and somatic embryos. On this medium, somatic embryos continuously develop into adventitious buds, which can form adventitious roots on a rooting medium containing NAA $(0.5 \mathrm{mg} / \mathrm{l})$. Meanwhile, on agar-solidified MS basal medium supplemented with BAP $(1 \mathrm{mg} / \mathrm{l})$ and NAA $(0.05 \mathrm{mg} / \mathrm{l})$, approximately $100 \%$ of explants from leaves \#3 - 6 form calli in less than 3 weeks of inoculation and adventitious buds via organogenesis in 3 - 4 weeks. In all six positional leaves, explants from the sixth leaf exhibit the rapidest response to induction of calli and adventitious buds. Nearly $100 \%$ adventitious buds can form adventitious roots on the rooting medium. Regenerated plants from both somatic embryogenesis and organogenesis complete self-pollination to produce seeds in 80 - 90 days of growth in growth chamber. LC-ESI-MS analysis demonstrates that regenerated plants biosynthesize artemisinin. These results show the highly efficient regeneration capacity of self-pollination A. annua plants that can form a new platform to enhance the understanding of artemisinin biosynthesis and metabolic engineering.
\end{abstract}

Keywords: Artemisia annua; Artemisinin; Biosynthesis; Self-Pollination; Somatic Embryogenesis; Organogenesis; HPLC-MS

\section{Introduction}

To date, Artemisia annua is the only natural resource producing artemisinin which is the main compound used in the artemisinin-based combination therapy (ACT) fighting against malarial diseases caused by parasites, such as Plasmodium falciparum and P. viva [1-5]. As we know, malaria is one of the most severe infectious diseases causing life loss of approximately one million people every year. Since 1970 s when artemisinin was identified to be an endoperoxide lactone sesquiterpene in A. апnиа by Chinese scientists [6,7], its medicinal activity helped Chinese people to effectively fight against and control

"Corresponding author. malarial disease in China. Later on, this medicine was recommended to other epidemic countries and regions by World Health Organization (WHO) $[1,3,8]$. Over the past years, due to the low and variable content of artemisinin in plants, its yield has never met the high demanding for therapy. To fight against malaria, both institutional laboratories and companies globally have started to investigate A. апnua and biosynthesis of artemisinin. Many great efforts have made multiple progresses in the areas of selection of ecotypes [9-11], genetic breeding $[2,5,11,12]$, tissue culture [2,13-15], genetic transformation [16-19], gene cloning and metabolic engineering [20-23]. Particularly, the biochemical and transgenic elucidation of biosynthetic steps from amorpha-4, 
11-diene to artemisinic acid [20,24,25] and dihyroartemisinic acid $[25,26]$ has provided a high potential for semi-synthesis of artemisinin. The introduction of these steps into yeast has allowed the production of artemisinic acid from fermentation [24]. This invention has developed a promising potential approach to synthesize artemisinin.

Currently, plant growth in the field is still the main approach to produce artemisinin for ACT of malaria. Over the past many years, breeding efforts have largely increased the yield of artemisinin [11,27] and enhanced the understanding of artemisinin biosynthesis [5,12]. However, due to the feature of $A$. аnnua preferring to cross pollination and hybridity of progenies $[5,27,28]$, the variation problem of artemisinin yield has remained to be resolved. In addition, no success in mutagenesis has been reported to use forward genetic tools to understand artemisinin biosynthesis. To overcome this problem, we have been endeavoring to breed self-pollination plants [2]. To date, we obtained F6 progenies of plants, in which no segregation occurs. Accordingly, this self-pollination population allows us being able to investigate genetics and regulation of artemisinin biosynthesis. Particularly, self-pollination plants will allow us continuing to use forward and reverse genetics to dissect the biosynthetic pathway of artemisinin and to use metabolic engineering approaches for high production.

As we know, a successful tissue culture system is the basis for genetic transformation. Over the past approximately 30 years, numerous experiments have been performed to use tissue culture to regenerate and propagate A. апnиа clones for artemisinin production, as a good result, basal medium and phytohormone combinations have been optimized for different ecotypes [13-15,29-36]. These past endeavors greatly helped us save time and labor to avoid testing all phytohormones. Therefore, in our investigation, we only selected a few of combinations of plants hormones to test regeneration capacity of self-pollination plants and develop protocols. Young seedlings were used as material resources. Leaves from the first node to the sixth node of seedlings were used as explants to compare their regeneration efficiency. Of them, the sixth leaf showed $100 \%$ efficiency in both somatic embryogenesis and organogenesis. This high regeneration efficiency allows us to further utilize selfpollination plants for future genetic transformation and knockout of genes to understand the biosynthetic pathway and regulation of artemisinin in the future.

\section{Materials and Methods}

\subsection{Chemicals}

Indo-3-butyric acid (IBA), naphthaleneacetic acid (NAA), 6-benzylaminopurine (BAP or 6-BA), sucrose, and phytoagar as well as chemicals of macronutrients, micronutrients and organic nutrients used in basal MS medium [37] were purchased from Plant Media (Dublin, OH, USA). Thidiazuron (TDZ) was purchased from Sigma (St. Louis, MO, USA).

\subsection{Medium Preparation and Photoperiod}

The basal MS medium was used in our experiments. Phytohormones used were sterilized using a filtration through a $0.2 \mu \mathrm{m}$ membrane. All media used in experiments were added $2 \%(\mathrm{~W} / \mathrm{V})$ sucrose and $0.45 \%(\mathrm{~W} / \mathrm{V})$ phytoagar, adjusted to $\mathrm{pH} 5.7$ with $1 \mathrm{~N} \mathrm{NaOH}$ and then autoclaved $35 \mathrm{~min}$ at $121^{\circ} \mathrm{C}$. After autoclaved, media were cooled down to $50^{\circ} \mathrm{C}-60^{\circ} \mathrm{C}$, necessary phytohormones were added to reach working concentrations used in each medium described below. Twenty milliliters of liquefied agar medium was poured into one petri dish (15 $\times 100 \mathrm{~mm}$, height $\times$ diameter) and then solidified at room temperature.

The photoperiod and temperature for callus induction and regeneration were set up at light/dark (16/8 hrs) and $24^{\circ} \mathrm{C}-25^{\circ} \mathrm{C}$, respectively. The light intensity was set up at $50 \mu \mathrm{mol} \cdot \mathrm{m}^{-1} \cdot \mathrm{s}^{-1}$.

\subsection{Seed Germination and Selection of Explants}

Seeds from self-pollination progeny plants (F5 and F6) of A. annua grown in phytotron were used in this experiment. Seeds were treated $1 \mathrm{~min}$ in $0.5 \mathrm{ml}$ of $70 \%$ ethanol contained in a sterile Eppendorf tube. During this treatment, the tube was vortexed thoroughly. Ethanol was then removed to a waste container. Seeds were subsequently washed four times using autoclaved deionized $\mathrm{H}_{2} \mathrm{O}$. These surface-sterilized seeds were then treated 5 min using 10\% Clorox in a sterile Eppendorf tube, during which the tube was vortexed $1 \mathrm{~min}$ thoroughly. After Clorox was disposed into a waste container, seeds were washed four times using autoclaved deionized $\mathrm{H}_{2} \mathrm{O}$. Sterilized seeds were placed on phytoagar-solidified MS medium contained in petri dishes, which were then placed in an incubator with necessary photoperiod and temperature described above.

After three weeks of seed germination, seedlings (Figure 1(a)) developed the first two true simple leaves (\#1 and \#2) in addition to the two cotyledons. The size of two leaves was approximately $0.8-1 \mathrm{~cm}$ in length. The first and second leaves of these three-week old seedlings were excised for explant materials. The $3^{\text {rd }}, 4^{\text {th }}, 5^{\text {th }}$ and $6^{\text {th }}$ leaves (Figure 1(b)) of 35-old seedlings were excised as explant materials.

For explant preparation, the first and second leaves were wounded on both adaxial and abaxial surfaces with 

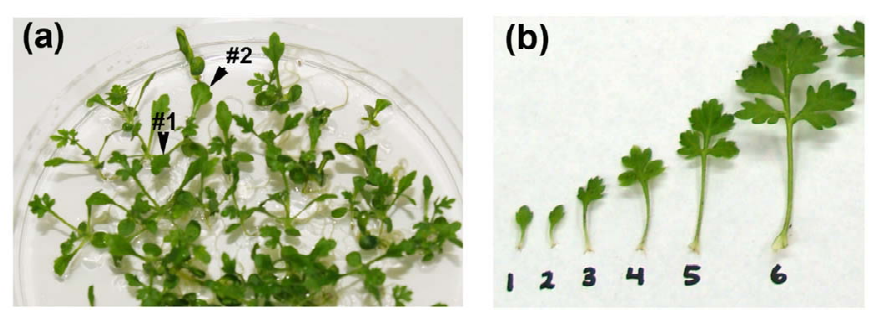

Figure 1. Growth of seedlings and leaves used for explants. (a) Seedlings (three-week old) grown on agar-solidified ME1 (basal MS) medium in a petri dish; (b) Morphologies of the $1^{\text {st }}$ and $2^{\text {nd }}$ leaves from three-week old seedlings and the $3^{\text {rd }}$ through $6^{\text {th }}$ leaves of seedlings from 35 -day old seedlings.

a sterilized razor. The $3^{\text {rd }}-6^{\text {th }}$ leaves were cut into approximately $0.8 \times 1 \mathrm{~cm}$ size pieces. Wounded leaf pieces were used as explants for induction of calli and adventitious buds described below.

\subsection{Treatment of Explants with TDZ and IBA Combinations}

Three different concentrations of TDZ and $0.1 \mathrm{mg} / 1 \mathrm{IBA}$ were selected to form three combinations (Table 1). Meanwhile, MS basal medium was used as a control. Thirty explants from the $1^{\text {st }}$ and $2^{\text {nd }}$ leaves (Figure 1(b)) were inoculated onto agar-solidified medium contained in one petri dish $(100 \times 15 \mathrm{~mm}$, diameter $\times$ height, in size). The other petri dish was performed as a technical repeat for each medium. Petri dishes were sealed with parafilm and placed under the culture condition described above. Explants were examined every day and taken pictures at different days (e.g. 2, 7, 17 and 30 days) after inoculation. The dates of callus and adventitious bud formation were recorded in detail. This experiment was repeated 4 times. In addition, this experiment was tested with both F5 and F6 progeny plants, respectively.

\subsection{Test of Regeneration Capacity among Leaves \#1 through \#6}

In this study, we selected two combinations of plant hormones to test regeneration capacity of explants from different positional leaves. One was $0.6 \mathrm{mg} / \mathrm{L} \mathrm{TDZ}$ and $0.1 \mathrm{mg} / \mathrm{L}$ IBA (ME4, Table 1) and the other was $1 \mathrm{mg} / \mathrm{l}$ BAP and $0.05 \mathrm{mg} / \mathrm{l} \mathrm{NAA}$.

Explants were obtained from $1^{\text {st }}, 2^{\text {nd }}, 3^{\text {rd }}, 4^{\text {th }}, 5^{\text {th }}$ and $6^{\text {th }}$ leaves (Figure 1(b)) respectively, of which explants from leaves \#1 and 2 were considered as one group, while each of others was as an individual group, respectively. Fifteen explants from each group were inoculated onto agar-solidified medium contained in one petri dish. Two petri dishes were tested as a technical repeat for each group of explants. Inoculation, observation and taking picture were as described above. This experiment was repeated 4 times and tested using both F5 and F6 progenies of plant, respectively.

\subsection{Induction of Adventitious Roots to Obtain Plantlets}

Based on many optimized media for rooting of adventitious buds/shoots reported previously (seeing discussion), we selected one medium consisting of basal MS medium supplemented with $0.05 \mathrm{mg} / \mathrm{l}$ NAA (Table 1). Agar-solidified rooting medium was contained in $15 \mathrm{~cm}$ long glass tubes.

Adventitious buds $(0.3-0.5 \mathrm{~cm}$ in length) from somatic embryos induced by TDZ and IBA combinations were excised from calli for root induction. Adventitious buds $(0.5-1 \mathrm{~cm}$ in length with 2 - 3 leaves) induced by BAP and NAA were separated from explants or calli for root induction. Adventitious buds were inoculated on rooting medium (ME5, Table 1) contained in glass tubes. All tubes were sealed with parafilm and placed under the culture condition described above.

\subsection{Plant Growth in Soil, Self-Pollination and Seed Germination}

After one month of root induction, plantlets were transplanted to small pots $(15 \times 15 \mathrm{~cm}$, diameter by height $)$ filled with premier Pro-Mix-PGX (fine granulated) soil. One pot was planted with one plantlet and then was placed on a nursery bed facilitated with a photoperiod of $12 / 12$ (light/dark) at $25^{\circ} \mathrm{C}$ in the Phytotron. The light intensity was set at $50 \mu \mathrm{E} / \mathrm{m}^{2} / \mathrm{sec}$. During the period in the nursery bed, plants were misted with tap water one time per $3 \mathrm{sec}$ during the light cycle and one time per 3 min during the dark cycle. After two weeks of growth, each regenerated plant was transplanted into a $10 \mathrm{~cm}$ pot filled with premier Pro-Mix-PGX (fine granulated) soil. All plants were then placed in a growth chamber facilitated with a photoperiod of $9 / 15 \mathrm{hrs}$ (light/dark). A temperature cycle was set at $26^{\circ} \mathrm{C} / 22^{\circ} \mathrm{C}$ (light/dark) as reported previously [2]. Plants were watered every other day with nutrients and alternate days with tap water.

To test self-pollination, each plant was covered using a plastic bag with an opening of the top and management of flowering and seed harvest were the same as reported previously [2]. 
Seeds harvested from regenerated plants were tested for germination on ME1 medium as described above. Seeds germinated on medium were recorded to evaluate the capacity of germination rate.

\subsection{Scanning Electron Microscope Observation of Somatic Embryogenesis}

After inoculation of 10, 12, 14, 16 and 18 days respectively, calli induced from explants on medium ME4 (Table 1) were collected for SEM observation. Calli were immersed in $3.0 \%$ glutaraldehyde dissolved in $0.05 \mathrm{M}$ potassium phosphate buffer $(\mathrm{pH} 6.6)$ at $4^{\circ} \mathrm{C}$. After two weeks, calli were washed with $0.05 \mathrm{M}$ potassium phosphate buffer ( $\mathrm{pH}$ 6.6), one change of buffer per $20 \mathrm{~min}$ for three changes at $4^{\circ} \mathrm{C}$. Washed calli were successively treated with $30 \%, 50 \%, 70 \%, 95 \%$ and $100 \%$ ethanol on ice, $2.5 \mathrm{hrs}$ per treatment, to remove water from calli. Dehydrated calli were warmed to room temperature. To obtain complete dehydration, calli were treated additional twice in $100 \%$ ethanol at room temperature, each $2 \mathrm{hrs}$. Dehydrated calli were summited to a critical point dry for 15 minutes at critical point using liquid carbon dioxide (Tousimis Samdri-795, Tousimis Research Corporation, Rockville, MD) and then were mounted on stubs with double-stick tape. Finally, mounted calli were sputter coated with approximately $50 \AA$ gold-palladium (Hummer 6.2 sputtering system, Anatech USA; Union City CA) and stored in a vacuum desiccator. Coated calli were scanned at $20 \mathrm{kV}$ using a JEOL JSM-5900LV (JEOL USA; Peabody, MA).

\subsection{Extraction of Artemisinin and LC-MS Analysis}

To understand if regenerated plants produce artemisinin, rosette leaves were collected from seedlings that were grown for 30 days in the photoperiod of $15 / 9 \mathrm{hrs}$ (light/dark). One hundred milligrams of fresh leaves was used to extract artemisinin using LC-MS grade methanol. Identification of artemisinin was carried out using LC-MS analysis on a 2010EV Shimadzu LC-PDA-ESI-

Table 1. Media tested for regeneration of self-pollinated $A$. annua progeny.

\begin{tabular}{cc}
\hline Medium & Components \\
\hline ME1 & $\begin{array}{c}\text { Basal MS medium solidified } \\
\text { with } 0.45 \% \text { phytoagar }\end{array}$ \\
ME2 (regeneration) & MS2 $+0.1 \mathrm{mg} / \mathrm{L} \mathrm{IBA}+0.2 \mathrm{mg} / \mathrm{L} \mathrm{TDZ}$ \\
ME3 (regeneration) & $\mathrm{MS} 2+0.1 \mathrm{mg} / \mathrm{L} \mathrm{IBA}+0.1 \mathrm{mg} / \mathrm{L} \mathrm{TDZ}$ \\
ME4 (regeneration) & $\mathrm{MS} 2+0.1 \mathrm{mg} / \mathrm{L} \mathrm{IBA}+0.6 \mathrm{mg} / \mathrm{L} \mathrm{TDZ}$ \\
ME5 (rooting) & $0.05 \mathrm{mg} / \mathrm{L} \mathrm{NAA}$ \\
\hline
\end{tabular}

MS instrumentation. Extraction and LC-MS analysis protocols were as described previously [2].

\section{Results}

\subsection{Induction of Embryogenic Calli, Somatic Embryos and Development of Plantlets}

In our experiments, before we tested other leaves, we firstly focused to use leaves \#1 and 2 (Figure 1(b)) to investigate effects of selected media on induction of callus and adventitious bud from explants. The reason was that experiments could be started after seed germination of three weeks. This method saved time. As described in methods, we investigated 4 media, ME1-ME4 (Table 1). The number of explants forming calli on each medium was recorded. In comparison, explants on ME4 showed the rapidest responses. In the first week, explants on this medium started to obviously expand to form calli from wounded sites. Approximately 3 weeks of inoculation, explants formed obvious friable yellow-greenish calli (Figure 2(a)) and developed a certain number of adven-
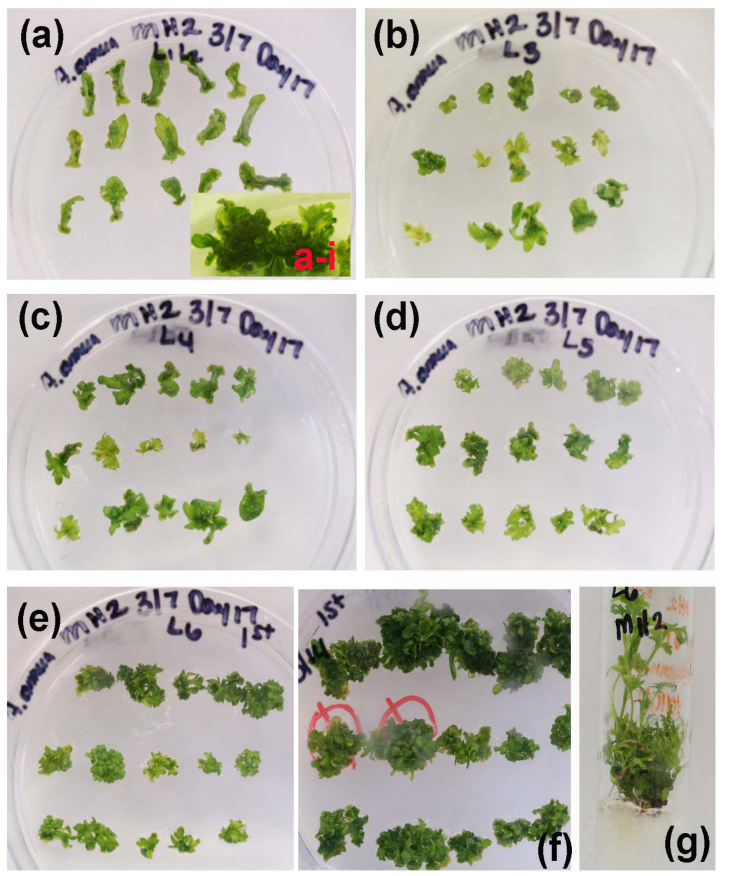

Figure 2. Regeneration from explants cultured on agarsolidified MS medium supplemented with TDZ (0.6 mg/l) and IBA (0.1 mg/l) (ME4, Table 1). Petri dish pictures (a)-(e) were taken after inoculation of explants for 17 days. (a) Explants from leaves \#1 and 2, in which "a-i" is an inserted image showing adventitious bud formation from calli at day 24 after inoculation of explants; (b)-(e) Explants from leaf \#3 (b), leaf \#4 (c), leaf \#5 (d) and leaf \#6 (e) show their differential responses; (f) Formation of adventitious buds from embryogenic calli induced from explants of leaf \#6 at day 24 after inoculation; (g) Plantlets obtained from rooting medium (ME5, Table 1). 
titious buds, which continuously developed leaf structures in the following $4^{\text {th }}$ and $5^{\text {th }}$ weeks of culture (Figure 2(a-i)). In addition, although unlike the rapid responses on ME4, yellow-greenish calli and adventitious buds were induced from explants on ME2 and ME3, respectively. In contrast, explants neither formed calli nor adventitious buds on ME1, the basal MS medium.

Among three combinations of TDZ and IBA (Table 1, ME2-ME3), the percentage of explants forming calli was similar on ME2, ME3 and ME4 after nearly 3 weeks of inoculation (Figure 3(a)). In contrast, the percentage of adventitious bud formation from calli was significantly lower on ME2 than on ME3 and ME4, between which the average value on ME4 was higher (Figure 3(a)). As a result, we used ME4 to compare regeneration capacities of leaves \#1 through 6. In contrast, neither calli nor adventitious buds were formed from explants on ME1 (Figure 3(a)).

To understand the features of calli, we collected callus samples induced on ME4 at different dates and then immediately fixed them for SEM observation. Under SEM, different stages of somatic embryo structures were observed, including globular, torpedo, heart and early cotyledon embryos (Figures 4(a)-(d)). These results demonstrated that TDZ and IBA tested induced embryogenic calli. The formation of adventitious buds on ME2-ME4 was via a procedure of somatic embryogenesis.

On ME4, somatic embryos could continuously develop into vegetative structures, such as buds and leaves (Figures 2(a-i) and (f)). Many adventitious buds with leaves were formed from calli after three weeks of induction. As culture continued, multiple independent adventitious shoots (elongated adventitious buds) became highly obvious. This result showed that on this medium, somatic embryos could further develop to form shoot apical meristems and leaves. Furthermore, this observation was highly obvious on explants from leaves \#3 - 6 described below. However, no plantlets were obtained on ME4. Neither was a plantlet formed on ME2 and ME3.

To obtain plantlets, adventurous shoots were inoculated onto a rooting medium (ME5, Table 1), which was supplemented with $0.1 \mathrm{mg} / \mathrm{l}$ NAA. On this medium, nearly $100 \%$ of shoots developed roots to form plantlets (Figure 2(g)). Therefore, the use of ME4 and ME5 were effective to induce regeneration via somatic embryogenesis.

\subsection{Comparison of Leaves \#1 through \#6 Responding to TDZ and IBA}

To understand regeneration capacity of different leaves from seedlings, we compared six positional leaves, including the $1^{\text {st }}$ and $2^{\text {nd }}$ leaves (leaves \#1 and 2) from three-week old seedlings and the $3^{\text {rd }}-6^{\text {th }}$ leaves (leaves
\#3 - 6) from five-week old seedlings. For this comparison, we tested explants on ME4. Responses of explants were recorded in detail at different dates. After inoculation of 17 days, almost all explants from different leaves formed embryogenic calli (Figures 2(a)-(e)); although the average percentage value of induction from leaves \#1 and 2 was slightly lower (Figure 3(b)).

The formation of adventitious buds (from somatic embryogenesis) was also obvious at day 17 after explant inoculation (Figures 2(a)-(e)). After three weeks of induction, multiple adventitious buds developed from somatic embryos were characterized with one-two leaves (Figures 2(a-i) and (f)) but without roots. In the six positional leaves, explants from the $6^{\text {th }}$ leaf exhibited the highest average percentage value (Figure 3(b)). As culture was extended to 3 - 4 weeks, embryogenic calli induced from all explants of the $6^{\text {th }}$ leaf formed multiple adventitious buds (Figure 2(f)).

Somatic embryos induced from different leaf explants could not form roots on ME4. For root induction, adventitious buds were cultured onto ME5, on which, nearly $100 \%$ of them formed roots to develop into complete plantlets (Figure 2(g)).

\subsection{Regeneration on Medium Supplemented with BAP and NAA}

Over the past 30 years, many concentration combinations of BAP and NAA were tested to induce organogenesis of A. annua plants. Multiple combinations of different phytohormones such as BAP, NAA, IAA, KT and IBA have been optimized for different ecotypes [13-15,34,35, 38-43]. Based on these previous reports, we only chose one combination consisting of $1 \mathrm{mg} / \mathrm{l} \mathrm{BAP}$ and $0.05 \mathrm{mg} / 1$ NAA to test regeneration capacity of leaves.

Leaves \#1 through \#6 of seedlings were used for explants to compare their responses to BAP and NAA. Resultant data showed differences in induction of both callus and adventitious bud among explants (Figure 3(c); Figures 5(a)-(e)). After inoculation of 17 days, all explants from leaves \#4, 5 and 6 formed greenish compact calli. The average induction rate of calli from leaf $\# 1$ and 2 was approximately $63 \%$, significantly lower than those values from other leaves (Figure 3(c)). As culture continued, all explants from different leaves produced calli. Under microscope, calli induced by BAP and NAA were relatively compact and different from embryogenic calli induced by TDZ and IBA described above.

In addition, of 6 positional leaves tested, explants from leaf \#6 gave the highest induction rate of adventitious bud formation at day 17, the average percentage value of which was significantly higher than those values from leaves \#1, 2, 3 and 4 (Figure 3(c)). Approximately 92\% of explants from leaf \#6 produced adventitious buds. 
(a)

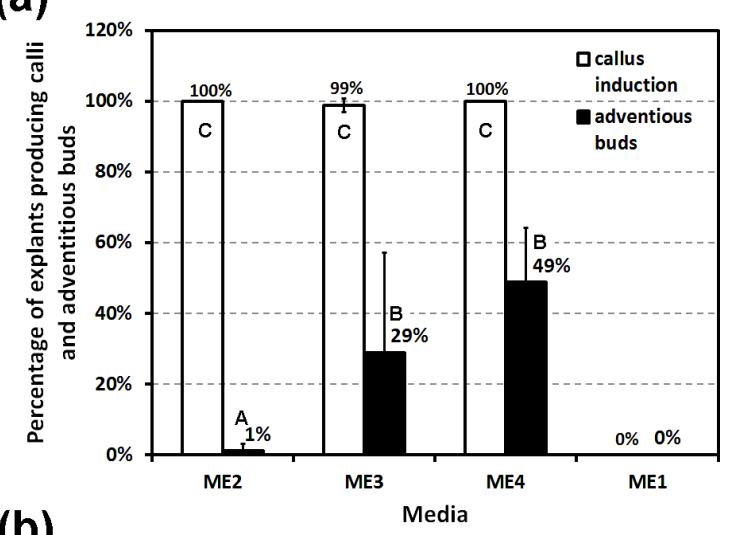

(b)

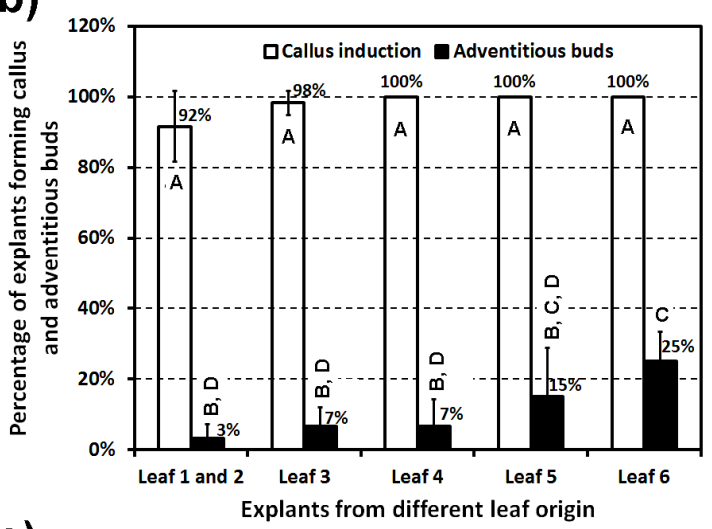

(c)

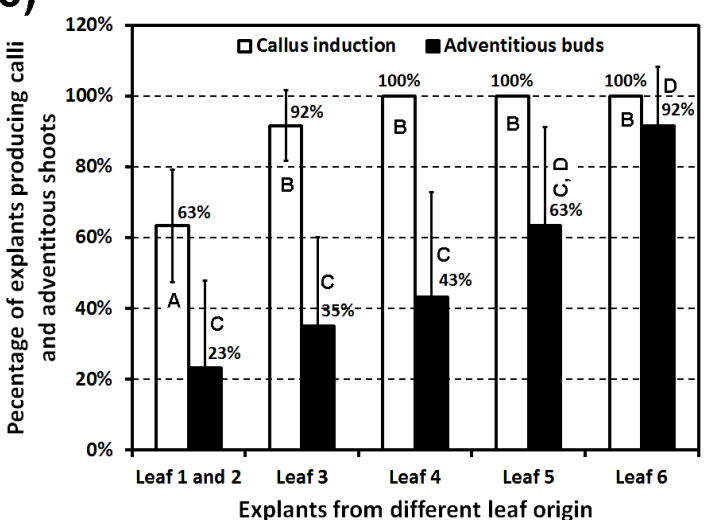

Figure 3. Effects of media and leaf positions on callus induction and adventitious bud formation. (a) Data show percentages of induction of embryogenic calli and formation of adventitious buds from leaf \#1 and 2 explants on ME1-ME4 (Table 1) after cultured 21 days; percentage values are mean values from 4 independent experiments, each of which was performed with 30 explants and technically repeated once; error bars are calculated from standard deviation. Columns labeled with different letters such as " $A$ " and " $B$ " indicate significant differences evaluated by Student's T-test, $P<0.05$, while labeled with the same letter such as " $B$ " and " $C$ " indicate insignificant differences. (b) Data show percentage values of embryogenic calli induction and formation of adventitious buds from explants of leaves \#1 - 6 after inoculation of 17 days on medium ME4; percentage values are mean values of 4 independent experiments, each of which was performed with 15 explants and technically repeated once; error bars were calculated from standard deviation. Columns labeled with the same capitalized letters indicate insignificant differences ( $P$ values $>0.05$ ), while columns labeled with different capitalized letters indicates significant differences $(P<0.05)$ evaluated by Student's $T$-test. (c) Data show percentage values of induction of calli and formation of adventitious buds from explants of leaves \#1 - 6 after cultured 17 days on MS medium supplemented with BAP (1 mg/l) and NAA (0.1 mg/l); percentage values are mean values of 4 independent experiments, each of which was performed with 15 explants and technically repeated once; standard error bars were calculated from standard deviation; columns labeled with the same capitalized letter(s) indicate insignificant differences $(P>0.05)$, while columns labeled with different capitalized letters indicates significant differences $(P<0.05)$ evaluated by Student's T-test. 


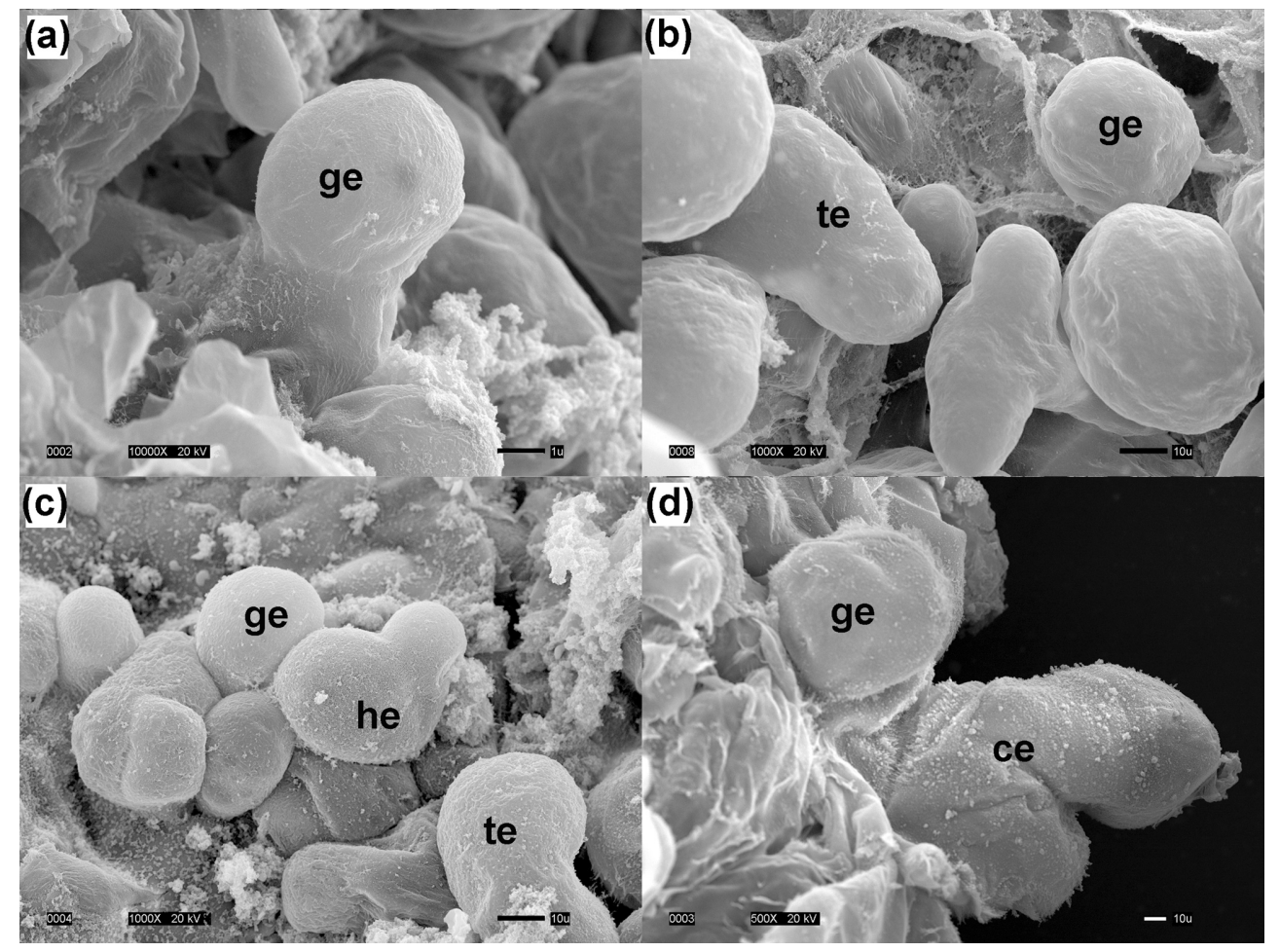

Figure 4. Images of scanning electron microscopy showing different stages of somatic embryo structures formed on embryogenic calli induced from explants of leaves \#1 and 2 on ME4. SEM images were taken from embryogenic calli induced from explants after inoculation of 10 (a), 12 - 14 (b), 16 (c) and 18 (d) days, respectively. ge: global embryos, te: torpedo embryos, he: heart embryos; ce: cotyledon embryos.

Furthermore, as culture continued, all explants of leaf \#6 produced multiple adventitious buds.

On this medium, adventitious buds could not develop roots. To induce roots, we cultured adventitious shoots on ME5. After three weeks of induction, approximately $100 \%$ of adventitious shoots formed roots to obtain plantlets (Figure 5(f)).

\subsection{Growth of Plantlets in Pot Soil, Self-Pollination and Seed Germination}

By following the growth protocol of seedlings, flowering induction and self-pollination that we developed previously [2], we grew regenerated plants in growth chamber to induce flowers and self-pollination. After plantlets were transplanted to small pots $(15 \times 15 \mathrm{~cm})$ filled with premier Pro-Mix-PGX (fine granulated) soil, most of them grew to develop new leaves and elongated stems in the photoperiod of 15/9 hrs (light/dark) in phytotron (Figure 6(a)). After nearly 5-week's growth in long photoperiod, regenerated plants were transferred into a growth chamber with a short photoperiod $(9 / 15 \mathrm{hrs}$, light/dark), in which plants started to develop flowers after additional two weeks of growth (Figure 6(b)) and then covered with sleeve-like plastic bag for self-pollination. All regenerated plants grew $25-35$ centers tall to start to bloom and set seeds (Figure 6(b)), as seedling growth reported previously.

After nearly 80 days of transplanting, plants were ready for seed harvest. Each pot containing one plant with numerous dry inflorescence heads was moved to a dry room, in which plants were not watered and naturally dried for one additional week at room temperature. Then, seeds (Figure 6(c)) were harvested from each individual plant and were used for germination test on ME1 medium. All mature seeds germinated to develop new seedlings in petri dishes. In addition, all seeds germinated in soil.

\subsection{LC-ESI-MS Analysis of Artemisinin}

The establishment of regeneration protocols was to investigate artemisinin biosynthesis in and accelerate metabolic engineering using self-pollination plants. In our experiments, artemisinin formation was investigated using LC-ESI-MS analysis. As reported previously to show artemisinin biosynthesis in self-pollinated plants, positive ionization mode was used to add one proton to artemisinin [2]. In this condition, artemisinin standard was created one main mass fragment, $341[\mathrm{~m} / \mathrm{z}]^{+}=[\operatorname{artemisinin}$ $+\mathrm{Na}+\mathrm{Cl}]^{+}$. In addition, another main mass fragment was created to be $305[\mathrm{~m} / \mathrm{z}]^{+}=$artemisinin $\left.+\mathrm{Na}\right]^{+}$. In 

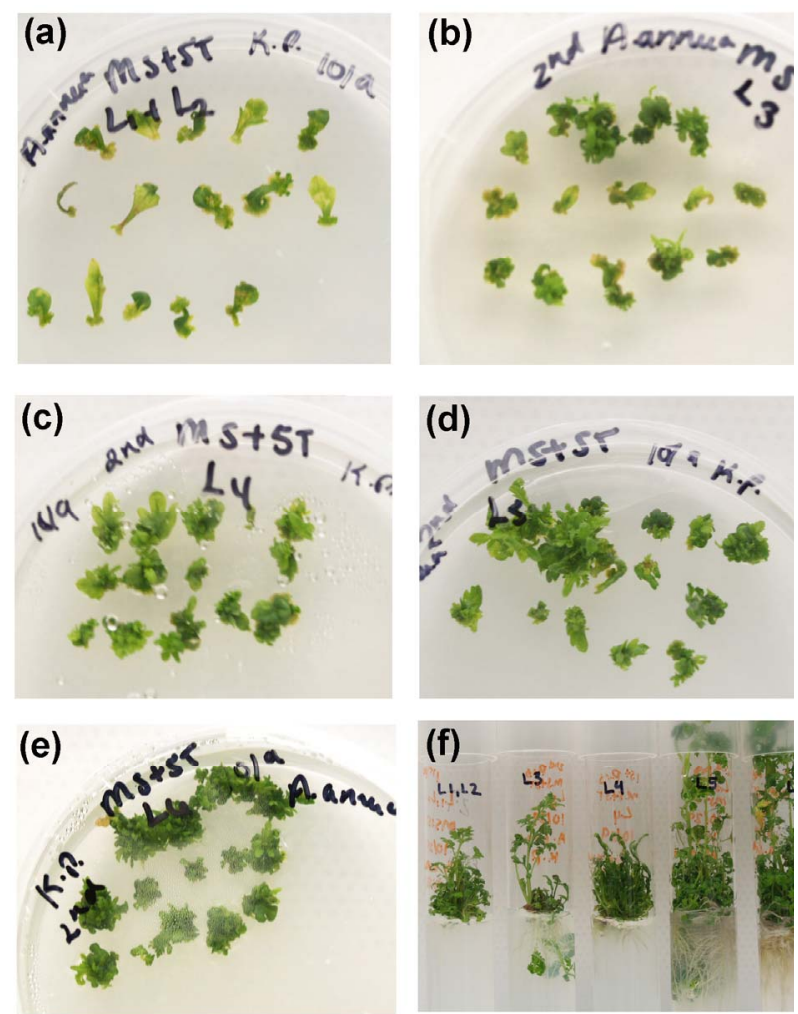

Figure 5. Regeneration from leaf explants on agar-solidified MS basal medium supplemented with BAP (1 mg/l) and NAA (0.1 mg/l). (a)-(e) Explants from leaves \#1 and 2 (a), leaf \#3 (b), leaf \#4 (c), leaf \#5 (d) and leaf \#6 (e), which were cultured 17 days, show their differential responses to this induction medium; (f) Plantlets obtained from rooting medium (ME5, Table 1).

our analysis, we used $341[\mathrm{~m} / \mathrm{z}]^{+}$to create selected ion chromatographs to detect artemisinin in extraction of leaf samples. As artemisinin standard showed, a high abundant peak of artemisinin was detected in leaf extracts at the same retention time (Figure $\mathbf{7 ( a ) )}$. However, this peak was not detected in root extracts (Figure 7(b)). This result demonstrated that regenerated plants produced artemisinin.

\section{Discussion}

In this study, our goal was to test regeneration capacity of seedling leaves and then develop an efficient regeneration protocol for self-pollination A. annua plants. We understood that in plant tissue culture, testing multiple combinations of plant hormones was essential to develop protocols, but, we did not follow this regular experimental logic in our experiments. The reason was that since middle 1980s, numerous experiments have been performed to use tissue culture to regenerate and propagate A. annua clones for artemisinin production [13-15,29-36]. Although there were many challenges in optimizing con-
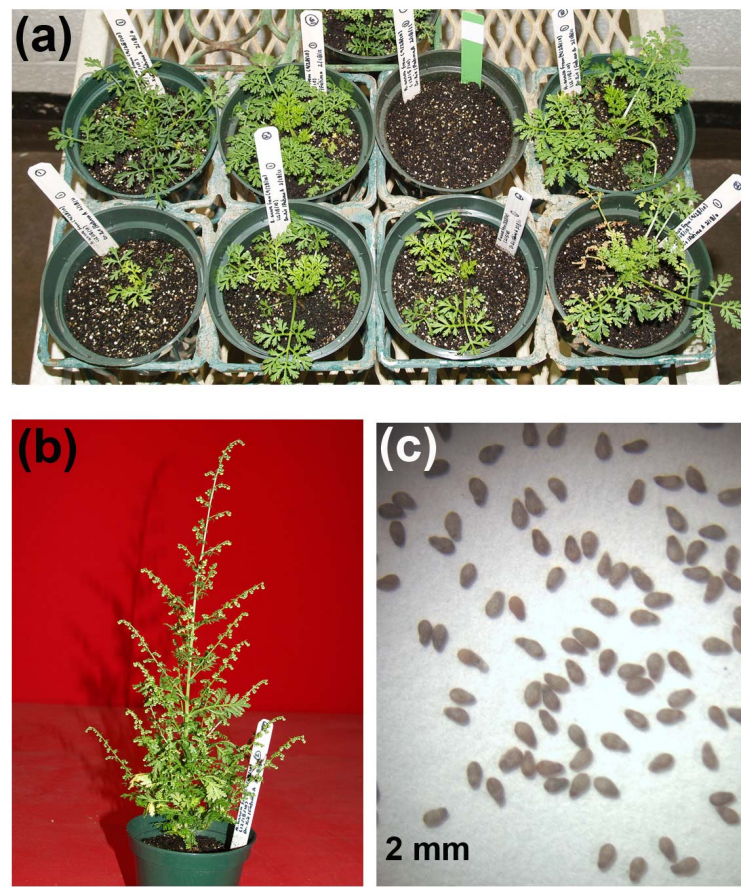

Figure 6. Growth, blooming and seeds of regenerated plants in growth chamber. (a) Examples of regenerated plants from somatic embryogenesis; (b) Blooming of a regenerated plant; (c) Seeds from self-pollination of a regenerated plant.

ditions for regeneration of different ecotypes of $A$. annua, numerous solid results regarding the use of basal medium and combinations of plant hormones such as 2, 4-D, BAP, NAA and IBA were obtained from those investigations. These useful data helped us save time and labor to avoid testing all of phytohormones. In comparison, TDZ was seldom used for regeneration of A. annua. Accordingly, we selectively tested 3 combinations consisting of TDZ and IBA (Table 1) and one combination of BAP and NAA to induce regeneration of self-pollination progenies and compare regeneration capacities of different leaves. The rationale to choosing TDZ was that this synthetic cytokinin has been reported to be able to induce somatic embryogenesis of many plants, such as Acacia mangium, Catharanthus roseus and Bambusa edulis [44-46]. In our experiments, results showed that $0.6 \mathrm{mg} / \mathrm{l} \mathrm{TDZ}$ and 0.1 $\mathrm{mg} / \mathrm{l}$ IBA highly efficiently induced somatic embryos from explants, particularly from leaf \#6 of seedlings (Figures 3(b) and 4), nearly $100 \%$ of which produced somatic embryos further forming adventitious buds. To our best knowledge, this is the first report to induce somatic embryogenesis from leaf explants of self-pollination plants of A. annua. In addition to somatic embryogenesis, the efficiency of organogenesis from leaves \#1 through 6 was very high (Figures 3(c) and 5). All explants from leaf \#6 produced adventitious buds in three weeks. These results demonstrated the high efficiency of 

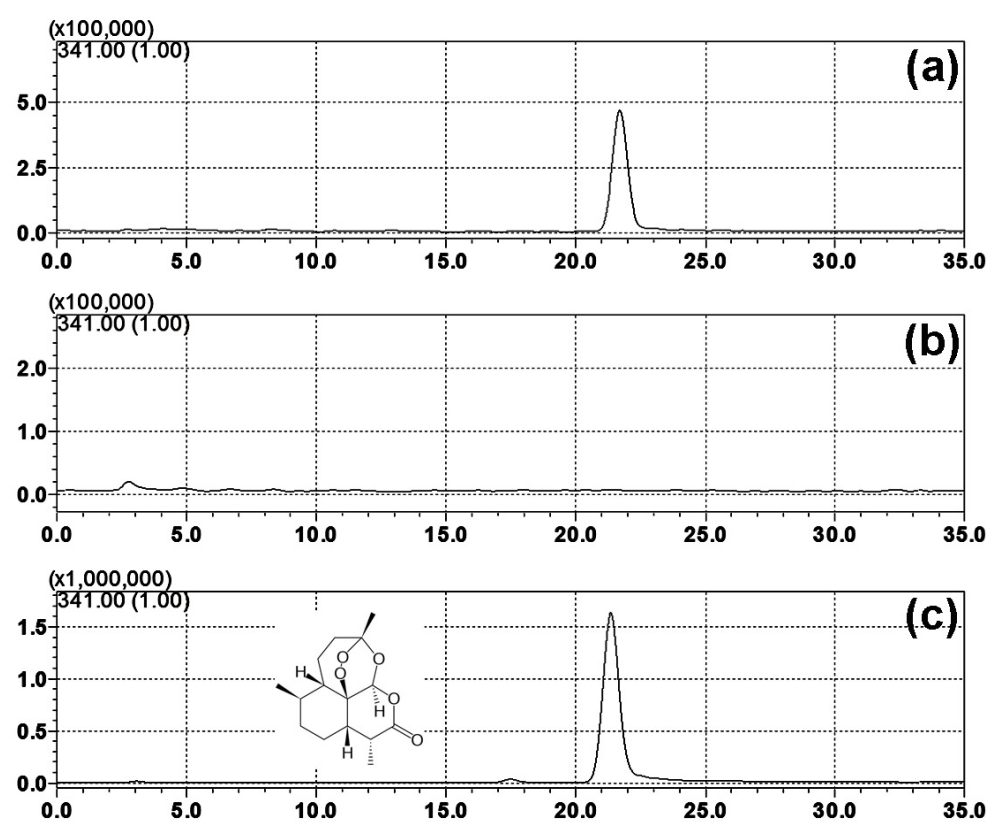

Figure 7. Selected ion chromatographs showing formation of artemisinin in leaves of regenerated plants. (a) A peak showing artemisinin in crude leaf extraction characterized by a mass-to-charge of $341[\mathrm{~m} / \mathrm{z}]^{+}$; (b) No peaks at 341 [m/z] $]^{+}$detected from crude root extraction; (c) An authentic standard of artemisinin characterized by a mass-to-charge of 341 [m/z] ${ }^{+}$.

regeneration capacity of leaves of self-pollination plants.

Another goal of this investigation was to compare regeneration capacity of different positional leaves of seedlings and to select explant resources for future genetic transformation to enhance metabolic engineering of artemisinin. We understand that testing all positional leaves can provide a comprehensive result showing regeneration capacities of all leaves. In consideration of reducing time, labor and spaces, we chose 3-week to 5 -week old seedlings grown on agar medium in our investigation. This time frame allowed us testing regeneration of explants in a relatively short period. Our data showed that although explants from the $1^{\text {st }}$ to the $6^{\text {th }}$ leaves of seedlings could efficiently produce embryogenic calli and adventitious buds on ME4, the average values of the $6^{\text {th }}$ leaf were higher than those of other leaves in nearly 3-week period after inoculation (Figure 3(b)). Actually, after continuous culture to 4 weeks, all explants of the $6^{\text {th }}$ leaf produced adventitious buds. In addition, on the medium containing BAP and NAA, explants from the $6^{\text {th }}$ leaf showed the highest percentage of adventitious bud induction in approximately 3 weeks after inoculation (Figure 3(c)). These results indicate that the $6^{\text {th }}$ leaf tested in our experiments has the highest regeneration capacity. The possible reason was that on 5 -week old seedlings, the $6^{\text {th }}$ leaf was less mature than others, thus gave the highest efficiency. The other possible reason was that the $6^{\text {th }}$ leaf itself had higher regeneration capacity than others. This is because the spatial positions of tissues have been reported to dramatically affect regeneration of plants, the examples of which are Рориlus trichocarpa [47], A. mangium [48] and Cornus canadensis [49].

This investigation is to develop self-pollination A. annua plants as a platform to understand genetics of artemisinin biosynthesis and to enhance metabolic engineering for high yield. As we know, the final elucidation of biosynthetic pathways of natural products essentially needs genetic evidence. For example, genetic evidence from Arabidopsis thaliana and other model plants has helped the intensive understanding of anthocyanin and proanthocyanidin pathways in the plant kingdom [50-54]. To date, biochemical, molecular and synthetic evidence has demonstrated the enzymatic steps from amorphor-4, 11-diene to artemisinic acid and dihydroartemisinic acid [22-26,55] and mapping of F1 hybrid of A. annua has helped identify loci associated with artemisinin formation [5], however, genetic evidence, such as knockout of genes and their impact on artemisinin productions, remains largely lacking. One of crucial reasons has been the challenge of the heterogeneous progeny resulting from the cross-hybridization preference of $A$. апnиa $[2,9,27,28]$. This heterogeneity of progeny increases difficulty to select mutant plants to identify pathway and regulatory genes involved in artemisinin biosynthesis. In addition, genetic transformation of $A$. апnиа has been a challenging hurdle in metabolic engineering of artemisinin most likely due to heterogeneity of progeny [43]. We have developed self-pollination plants [2]. Progenies of F5 and F6 generations have not shown any segregation in 
plant growth and morphology as well as artemisinin formation, indicating that they are mostly likely inbred homozygous plants. We believe that the protocol of efficient regeneration developed in present study will help accelerate the use of self-pollination plants to develop genetic approaches such as mutagenesis to elucidate biosynthetic steps and regulatory mechanism of artemisinin formation.

\section{Conclusion}

The high regeneration variation of different ecotypes of $A$. annua plants has been reported to be a severe hurdle for the success of genetic transformation. The main reason likely is the segregation of progeny plants resulting from natural cross-hybridization. Our experiments demonstrate a high and reproducible regeneration efficiency of selfpollinated A. annua progeny through both somatic embryogenesis and organogenesis. Positional effects of leaves from juvenile seedlings on callus induction and regeneration are observed in our experiments. In the selected first six leaves of seedlings, the sixth leaf shows the rapidest response to induction of embryogenic callus and organogenesis as well as regeneration. Regenerated plants from both somatic embryogenesis and organogenesis produced a valuable level of artemisinin. Our data show that self-pollinated A. апnиa plants form an appropriate platform to genetically understand artemisinin biosynthesis and enhance metabolic engineering.

\section{Acknowledgements}

This research was funded by Multidisciplinary Research Grant (MRG) Program at North Carolina Biotechnology Center. We are grateful to Mrs. Valerie Knowlton at Center for Electron Microscopy for her assistance in SEM.

\section{REFERENCES}

[1] WHO, "Artemisinin and Its Derivatives as Anti-Malarial Drugs," WHO, Geneva, 1998.

[2] F. Alejos-Gonzalez, G. S. Qu, L. L. Zhou, C. H. Saravitz, J. L. Shurtleff and D.-Y. Xie, "Characterization of Development and Artemisinin Biosynthesis in Self-Pollinated Artemisia annua Plants," Planta, Vol. 234, No. 4, 2011, pp. 685-697.

[3] WHO, "Meeting on the Production of Artemisinin and Artemisinin-Based Combination Therapies," WHO, Arusha, 2006

[4] WHO, "Good Procurement Practices for ArtemisininBased Antimalarial Medicines," WHO Global Malaria Programme, WHO, Geneva, 2010.

[5] I. A. Graham, K. Bessr, S. Blumer, C. A. Branigan, T. Czechowski, L. Elias, I. Guterman, D. Harvey, P. G. Isaac,
A. M. Khan, T. R. Larson, Y. Li, T. Pawson, T. Penfield, M. F. Smallwood, V. Segura, T. Townsend, D. Vyas, T. Winzer and D. Bowles, "The Genetic Map of Artemisia annua L. Identifies Loci Affecting Yield of the Antimalarial Drug Artemisinin," Science, Vol. 327, No. 5963, 2010, pp. 328-331.

http://dx.doi.org/10.1126/science.1182612

[6] J.-M. Liu, M.-Y. Ni, J-F. Fan, Y.-Y. Tu, Z.-H. Wu and Y.-L. Wu, "Structure and Reaction of Arteannuin," Acta Chimica Sinica, Vol. 37, No. 2, 1979, pp. 129-143.

[7] Zhong-Yi-Yan-Jiu-Yue-Zhong-Yao-Yan-Jiu-Suo, "Antimalarial Studies of Artemisia annua L:1971-1978," Zhong Yi Yan Jiu Yue Zhong Yao Yan Jiu Suo, Beijing, 1978 .

[8] WHO, "The Role of Artemisinin and Its Derivatives in the Current Treatment of Malaria (1994-1995)," Unpublished Document, WHO/MAL/94.1067, World Health Organization, Geneva, 1994.

[9] J. F. S. Ferreira, "Cultivation and Genetics of Artemisia annua L. for Increased Production of the Antimalarial Artemisinin," Plant Genetic Resources: Characterization and Utilization, Vol. 3, No. 2, 2005, pp. 206-229. http://dx.doi.org/10.1079/PGR200585

[10] E. V. Geldre, A. Vergauwe and E. V. den Eeckhout, "State of the Art of the Production of the Antimalarial Compound Artemisinin in Plants," Plant Molecular Biology, Vol. 33, No. 2, 1997, pp. 199-209. http://dx.doi.org/10.1023/A:1005716600612

[11] J. Cockram, C. Hill, C. Burns, R. R. J. Arroo, J. G. Woolley, I. Flockart, T. Robison, C. J. Atkinson, M. J. Davies, N. Dungey, A. J. Greenland, L. Smith and S. Bentley, "Screening a Diverse Collection of Artemisia annua Germplasm Accessions for the Antimalarial Compound, Artemisinin," Plant Genetic Resources, Vol. 10, No. 2, 2012, pp. 152-154. http://dx.doi.org/10.1017/S1479262112000159

[12] T. E. Wallaart, N. Pras and W. J. Quax, "Seasonal Variations of Artemisinin and Its Biosynthetic Precursors in Tetraploid Artemisia annua Plants Compared with the Diploid Wild-Type," Planta Medica, Vol. 65, No. 8, 1999, pp. 723-728. http://dx.doi.org/10.1055/s-1999-14094

[13] M. S. R. Nair, N. Acton, D. L. Klayman, K. Kendrick, D. V. Basile and S. Mante, "Production of Artemisinin in Tissue Cultures of Artemisia annua," Journal of Natural Products, Vol. 49, No. 3, 1986, pp. 504-507.

[14] D. V. Basile, N. Akhtari, Y. Durand and M. S. R. Nair, "Toward the Production of Artemisinin through Tissue Culture: Nutrient-Hormone Combinations Suitable for Cell Suspension Cultures," In Vitro Cellular and Developmental Biology: Plant, Vol. 29, No. 3, 1993, pp. 143147. http://dx.doi.org/10.1007/BF02632286

[15] J. F. S. Ferreira, J. E. Simon and J. Janick, "Relationship of Artemisinin Content of Tissue-Cultured, Greenhouse, and Field-Grown Plants of Artemisia annua," Planta Medica, Vol. 61, No. 4, 1995, pp. 351-355. http://dx.doi.org/10.1055/s-2006-958098

[16] G. Cai, G. Li, H. Ye and G. F. Li, "Hairy Root Culture of Artemisia annua L. by Ri Plasmid Transformation and 
Biosynthesis of Artemisinin," Chinese Journal of Biotechnology, Vol. 11, No. 4, 1995, pp. 227-235.

[17] D.-Y. Xie, H. Ye, G. Li and Z. Guo, “Artemisia annua L. Transformation with Different Agrobacterium rhizogenesis and Large Scale Culture of Hairy Roots for Artemisinin (Qinghaosu) Production," In: Agricultural Biotechnology: Laboratory, Field and Market, CPN Publications, Darwin, 1998, pp. 134-136.

[18] S. Banerjee, M. Zehra and M. M. Kumar, "Agrobacterium rhizogenes-Mediated Transformation of Artemisia annua: Production of Transgenic Plants," Planta Medica, Vol. 63, No. 5, 1997, pp. 467-469.

http://dx.doi.org/10.1055/s-2006-957737

[19] B. Ghost, S. Mukherjee and S. Jha, "Genetic Transformation of Artemisia annua by Agrobacterium tumefaciens and Artemisinin Synthesis in Transformed Cultures," Plant Science, Vol. 122, No. 2, 1997, pp. 193-199. http://dx.doi.org/10.1016/S0168-9452(96)04558-X

[20] K. H. Teoh, D. R. Polichak, D. W. Reed, G. Nowak and P. S. Covello, "Artemisia annua L. (Asteraceae) TrichomeSpecific cDNAs Reveal CYP71AV1, a Cytochrome P450 with a Key Role in the Biosynthesis of the Antimalarial Sesquiterpene Lactone Artemisinin," FEBS Letters, Vol. 580, No. 5, 2006, pp. 1411-1416. http://dx.doi.org/10.1016/j.febslet.2006.01.065

[21] L. Zhang, F. Y. Jing, F. P. Li, M. Y. Li, Y. L. Wang, G. F. Wang, X. F. Sun and K. X. Tang, "Development of transgenic Artemisia annua (Chinese Wormwood) Plants with an Enhanced Content of Artemisinin, an Effective AntiMalarial Drug, by Hairpin-RNA-Mediated Gene Silencing," Biotechnology Applied Biochemistry, Vol. 52, No. 3, 2009, pp. 199-207. http://dx.doi.org/10.1042/BA20080068

[22] H. J. Bouwmeester, T. E. Wallaart, M. H. A. Jansen, B. van Loo, B. J. M. Jansen, M. A. Posthumus, C. O. Schmidt, J.-W. Kraker, W. A. Konig and M. C. R. Franssen, "Amorpha-4,11-Diene Synthase Catalyses the First Probable Step in Artemisinin Biosynthesis," Phytochemistry, Vol. 52, No. 5, 1999, pp. 843-854. http://dx.doi.org/10.1016/S0031-9422(99)00206-X

[23] P. S. Covello, K. H. Teoh, D. R. Polichuk, D. W. Reed and G. Nowak, "Functional Genomics and the Biosynthesis of Artemisinin," Phytochemistry, Vol. 68, No. 14, 2007, pp. 1864-1971. http://dx.doi.org/10.1016/j.phytochem.2007.02.016

[24] D.-K. Ro, E. M. Paradise, M. Oueller, K. J. Fisher, K. L. Newman, J. M. Ndungu, K. A. Ho, R. A. Eachus, T. S. Ham, J. Kirby, M. C. Y. Chang, S. T. Withers, Y. Shiba, R. Sarpong and J. D. Keasling, "Production of the Antimalarial Drug Precursor Artemisinic Acid in Engineered Yeast," Nature, Vol. 440, No. 7086, 2006, pp. 940-943. http://dx.doi.org/10.1038/nature04640

[25] Y. Zhang, K. H. Teoh, D. W. Reed and P. S. Covello, "Molecular Cloning and Characterization of Dbr1, a 2Alkenal Reductase from Artemisia annua," Botany, Vol. 87, No. 6, 2009, pp. 643-649. http://dx.doi.org/10.1139/B09-033

[26] Y. Zhang, K. H. Teoh, D. W. Reed, L. Maes, A.
Goossens, D. J. H. Olson, A. R. S. Ross and P. S. Covello, "The molecular Cloning of Artemisinic Aldehyde $\Delta-11(13)$ Reductase and Its Role in Glandular Trichome-Dependent Biosynthesis of Artemisinin in Artemisia annua," Journal Biological Chemistry, Vol. 283, No. 31, 2008, pp. 2150121508. http://dx.doi.org/10.1074/jbc.M803090200

[27] N. Delabays, X. Simonnet and M. Gaudin, "The Genetics of Artemisinin Content in Artemisia annua L. and the Breeding of High Yielding Cultivars," Current Medicinal Chemistry, 2001. Vol. 8, No. 15, 2001, pp. 1795-1801.

[28] N. Delabays, A. Benakis and G. Collet, "Selection and Breeding for High Artemisinin (Qinghaosu) Yielding Strains of Artemisia annua," Acta Horticulture, Vol. 330, No. 1, 1993, pp. 203-207.

[29] D.-Y. Xie, H. Ye and G. Li, "The Progress of Artemisia annua Research-The Application of Biotechnology and Prospects," Chinese Bulletin Botany, Vol. 12, No. 4, 1995 , pp. 28-31. (in Chinese)

[30] H. J. Woerdenbag, N. Pras and A. W. Alfermann, "Production of Artemisinin in Shoot Cultures of Artemisia annua," Planta Medica, Vol. 57, 1991, pp. A91-A92. http://dx.doi.org/10.1055/s-2006-960369

[31] H. M. Elhag, M. M. El-Domiaty, F. S. El-Olemy, J. S. Mossa and M. M. El-Olemy, "Selection and Micropropagation of High Artemisinin Producing Clones of Artemisia annua L.," Phytotherapy Research, Vol. 6, No. 1, 1992, pp. 20-24. http://dx.doi.org/10.1002/ptr.2650060106

[32] H. J. Woerdenbag, J. F. J. Lüers, Wv. Uden, N. Pras, T. M. Malingré and A. W. Alfermann, "Production of the New Antimalarial Drug Artemisinin in Shoot Cultures of Artemisia annua," Planta Medica, Vol. 58, No. S1, 1992, pp. 620-621. http://dx.doi.org/10.1055/s-2006-961621

[33] H. J. Woerdenbag, J. F. J. Luers, W. Vanuden, N. Pras, T. M. Malingre and A. W. Alfermann, "Production of the New Antimalarial Drug Artemisinin in Shoot Cultures of Artemisia annua L," Plant Cell, Tissue, and Organ Culture, Vol. 32, No. 2, 1993, pp. 247-257. http://dx.doi.org/10.1007/BF00029850

[34] N. B. Paniego and A. M. Giulietti, "Artemisia annua L.: Dedifferentiated and differentiated cultures," Plant Cell, Tissue, and Organ Culture, Vol. 36, No. 2, 1994, pp. 163168. http://dx.doi.org/10.1007/BF00037715

[35] A. Gulati, S. Bharel, S. K. Jain, M. Z. Abdin and P. S. Srivastava, "In Vitro Micropropagation and Flowering in Artemisia annua," Journal of Plant Biochemistry and Biotechnology, Vol. 5, No. 1, 1996, pp. 31-35. http://dx.doi.org/10.1007/BF03262976

[36] W. Lualon, W. De-Eknamkul, H. Tanaka, Y. Shoyama and W. Putalun, "Artemisinin Production by Shoot Regeneration of Artemisia annua L. Using Thidiazuron," Zeitschrift für Naturforschung C, Vol. 63, No. 1/2, 2008, pp. 96-100.

[37] T. Murashige and F. Skoog, "A Revised Medium for Rapid Growth and Bioassays with Tobacco Tissue Culture," Physiologia Plantarum, Vol. 15, No. 3, 1962, pp. 473-497. http://dx.doi.org/10.1111/j.1399-3054.1962.tb08052.x 
[38] K. L. Chan, C. K. H. Teo, S. Jinadasa and K. H. Yuen, "Selection of High Artemisinin Yielding Artemisia annua," Planta Medica, Vol. 61, No. 3, 1995, pp. 285-287. http://dx.doi.org/10.1055/s-2006-958078

[39] D. C. Jain, A. K. Mathur, M. M. Gupta, A. K. Singh, R. K. Verma, A. P. Gupta and S. Kumar, "Isolation of High Artemisinin-Yielding Clones of Artemisia annua," Phytochemistry, Vol. 43, No. 5, 1996, pp. 993-1001. http://dx.doi.org/10.1016/S0031-9422(96)00369-X

[40] A. Vergauwe, R. Cammaert, D. Vandenberghe, C. Genetello, D. Inzé, M. Montagu and E. Eeckhout, "Agrobacterium tumefaciens-Mediated Transformation of Artemisia annua L. and Regeneration of Transgenic Plants," Plant Cell Reports, Vol. 15, No. 12, 1996, pp. 929-933. http://dx.doi.org/10.1007/BF00231590

[41] A. Vergauwe, E. Van Geldre, D. Inzé, M. Van Montagu and E. Van den Eeckhout, "Factors Influencing Agrobacterium tumefaciens-Mediated Transformation of Artemisia annua L.," Plant Cell Reports, Vol. 18, No. 1, 1998, pp. 105-110. http://dx.doi.org/10.1007/s002990050540

[42] J.-L. Han, H. Wang, H.-C. Ye, Y. Liu, Z.-Q. Li, Y. Zhang, Y.-S. Zhang, F. Yan and G.-F. Li, "High Efficiency of Genetic Transformation and Regeneration of Artemisia annua L. via Agrobacterium tumefaciens-Mediated Procedure," Plant Science, Vol. 168, No. 1, 2005, pp. 73-80. http://dx.doi.org/10.1016/j.plantsci.2004.07.020

[43] B. Y. Liu, H. Wang, Z. G. Du, G. F. Li and H. C. Ye, "Metabolic Engineering of Artemisinin Biosynthesis in Artemisia annua L.," Plant Cell Reports, Vol. 30, No. 5, 2011, pp. 689-694. http://dx.doi.org/10.1007/s00299-010-0967-9

[44] D.-Y. Xie and Y. Hong, "Regeneration of Acacia mangium through Somatic Embryogenesis," Plant Cell Reports, Vol. 20, No. 1, 2001, pp. 34-40. http://dx.doi.org/10.1007/s002990000288

[45] C.-C. Lin, C.-S. Lin and W.-C. Chang, "In Vitro Flowering of Bambusa edulis and Subsequent Plantlet Survival," Plant Cell, Tissue and Organ Culture, Vol. 2, No. 1, 2003, pp. 71-78. http://dx.doi.org/10.1023/A:1021281217589

[46] M. Dhandapani, D. Kim and S.-B. Hong, "Efficient Plant Regeneration via Somatic Embryogenesis and Organogenesis from the Explants of Catharanthus roseus," In Vitro Cellular \& Developmental Biology: Plant, Vol. 44, No. 1, 2008, pp. 18-25. http://dx.doi.org/10.1007/s11627-007-9094-x
[47] J. Y. Song, S. F. Lu, Z. Z. Chen, R. Lourenco and V. L. Chian, "Genetic Transformation of Populus trichocarpa Genotype Nisqually-1: A Functional Genomic Tool for Woody Plants," Plant Cell Physiology, Vol. 47, No. 11, 2006, pp. 1582-1589.

[48] D.-Y. Xie and Y. Hong, "Agrobacterium-Mediated Genetic Transformation of Acacia mangium," Plant Cell Reports, Vol. 20, No. 10, 2002, pp. 917-922. http://dx.doi.org/10.1007/s00299-001-0397-9

[49] C.-M. Feng, R. Qu, L.-L. Zhou and D.-X. Xie, "Shoot regeneration of dwarf dogwood (Cornus canadensis L.) and Morphological Characterization of the Regenerated Plants," Plant Cell, Tissue and Organ Culture, Vol. 97, No. 1, 2009, pp. 27-37. http://dx.doi.org/10.1007/s11240-009-9495-0

[50] D.-Y. Xie and R. A. Dixon, "Proanthocyanidin Biosynthesis-Still More Questions than Answers?" Phytochemistry, Vol. 66, No. 18, 2005, pp. 2127-2144. http://dx.doi.org/10.1016/j.phytochem.2005.01.008

[51] R. A. Dixon, D.-Y. Xie and S. B. Sharma, "Proanthocyanidins-A Final Frontier in Flavonoid Research?" New Phytologist, Vol. 165, No. 1, 2005, pp. 9-28. http://dx.doi.org/10.1111/j.1469-8137.2004.01217.x

[52] D.-Y. Xie, S. B. Sharma, N. L. Paiva, D. Ferreira and R. A. Dixon, "Role of Anthocyanidin Reductase, Encoded by BANYULS in Plant Flavonoid Biosynthesis," Science, Vol. 299, No. 5605, 2003, pp. 396-399. http://dx.doi.org/10.1126/science. 1078540

[53] Kakegawa, K. Kakegawa, J. Suda, M. Sugiyama and A. Komamine, "Regulation of Anthocyanin Biosynthesis in Cell Suspension Cultures of Vitis in Relation to Cell Division," Physiologia Plantarum, Vol. 94, No. 4, 1995, pp. 661-666. http://dx.doi.org/10.1111/j.1399-3054.1995.tb00981.x

[54] B. Winkel-Shirley, "Flavonoid Biosynthesis. A Colorful Model for Genetics, Biochemistry, Cell Biology, and Biotechnology," Plant Physiology, Vol. 126, No. 2, 2001, pp. 485-493. http://dx.doi.org/10.1104/pp.126.2.485

[55] K. H. Teoh, D. R. Polichuk, D. W. Reed and P. S. Covello, "Molecular Cloning of an Aldehyde Dehydrogenase Implicated in Artemisinin Biosynthesis in Artemisia annua," Botany, Vol. 87, No. 6, 2009, pp. 635-642. http://dx.doi.org/10.1139/B09-032 